\title{
Selecting Time Series Length to Moderate the Impact of Nonstationarity in Extreme Rainfall Analyses
}

\author{
Arthur T. DeGaetano and Christopher Castellano \\ Northeast Regional Climate Center, Department of Earth and Atmospheric Science, Cornell University, Ithaca, New York
}

(Manuscript received 13 April 2018, in final form 9 August 2018)

\begin{abstract}
Observed and projected increases in the frequency of extreme rainfall complicate the extreme value analyses of precipitation that are used to guide engineering design specifications, because conventional methods assume stationarity. Uncertainty in the magnitude of the trend in future years precludes directly accounting for the trend in these analyses. While previous extreme value analyses have sought to use as long a record as possible, it is shown using stochastically generated time series that this practice exacerbates the potential error introduced by long-term trends. For extreme precipitation series characterized by a trend in the location parameter exceeding approximately $0.005 \% \mathrm{yr}^{-1}$, limiting the record length to fewer than 70 years is recommended. The use of longer time periods results in partial-duration series that are significantly different from their stationary counterparts and a greater percentage of rainfall extremes that exceed the $90 \%$ confidence interval corresponding to a stationary distribution. The effect is most pronounced on the shortest (i.e., $2 \mathrm{yr}$ ) recurrence intervals and generally becomes undetectable for recurrence intervals of more than 25 years. The analyses also indicate that the practice of including stations with records of limited length that end several decades prior to the present should be avoided. Distributions having a stationary location parameter but trended scale parameter do not exhibit this behavior.
\end{abstract}

\section{Introduction}

Extreme precipitation climatologies are an important component of engineering design. The frequency of large precipitation events provides the foundation for regulations and design specifications for infrastructure that must withstand or protect against such extreme events. These analyses provide critical information that is used in a wide range of applications, including urban and rural development, public infrastructure, watershed management, agriculture, and human health (e.g., Hailegeorgis and Alfredsen 2017; van der Pol et al. 2015). Through the years, in the United States there has been a sequence of analyses examining and updating the extreme rainfall climatology. These analyses include, most notably, Hershfield (1961), a series of regional atlases published in the 1990s (e.g., Huff and Angel 1992; Wilks and Cember 1993), and, most recently, a more comprehensive set of regional atlases covering most of the United States (e.g., Perica et al. 2013). Each has followed the same broad method, that is, extraction of the largest observed precipitation totals from a station's record, fitting of a

\footnotetext{
Corresponding author: Dr. Art DeGaetano, atd2@cornell.edu
}

theoretical extreme value distribution to these data, and calculation of precipitation amounts corresponding to specific percentiles of the fit distribution.

Another commonality has been the assumption of a stationary precipitation record. While valid in some regions, in others the historical record has been characterized by significant increases in extreme precipitation since the mid-to-late twentieth century (DeGaetano 2009; Groisman et al. 2012; Heineman 2012; Kunkel et al. 1999; Kunkel 2003). This has raised practical concerns about use of an assumed stationary historical record to design infrastructure with a lifetime that extends well into the future. This problem is exacerbated by climate model projections suggesting that the frequency and magnitude of extreme precipitation will continue to increase throughout the twenty-first century (e.g., IPCC 2014; Donat et al. 2016; Ning et al. 2015; Sun et al. 2016). As a result, stakeholders in many extreme rainfall-sensitive sectors have adopted various approaches to address these expected changes, including the addition of a safety factor to the historical $1 \%$ annual recurrence value, substitution of the historical $0.2 \%$ annual recurrence value for the $1 \%$ value (M. Lowery 2016, personal communication), and consideration of downscaled 
TABLE 1. Period of record (yr), GEV distribution parameters $(\mathrm{cm})$, and precipitation amounts $(\mathrm{cm})$ corresponding to the 2-, 50-, and 100 -yr average recurrence interval.

\begin{tabular}{|c|c|c|c|c|c|}
\hline & Phoenix & Seattle & Ithaca & Des Moines & Miami \\
\hline Record length & 122 & 123 & 112 & 140 & 107 \\
\hline \multicolumn{6}{|l|}{ GEV parameters } \\
\hline Location & 2.98 & 4.58 & 5.22 & 6.42 & 12.16 \\
\hline Scale & 0.51 & 0.69 & 0.77 & 0.88 & 2.75 \\
\hline Shape & -0.32 & -0.29 & -0.35 & -0.26 & -0.32 \\
\hline \multicolumn{6}{|l|}{ Precipitation amount } \\
\hline 2-yr recurrence & 3.17 & 4.85 & 5.52 & 6.76 & 13.23 \\
\hline 50 -yr recurrence & 6.94 & 9.63 & 11.67 & 12.36 & 33.60 \\
\hline $100-y r$ recurrence & 8.34 & 11.31 & 14.06 & 14.21 & 41.15 \\
\hline
\end{tabular}

climate model projections of future rainfall extremes (e.g., Wang et al. 2015; Rodríguez et al. 2014; DeGaetano and Castellano 2017). Other approaches, such as directly incorporating a trend term in the fit extreme value distribution (e.g., Katz et al. 2002; Cheng et al. 2014), have been proposed but require an assumption about the character of the trend beyond the available data record.

A third commonality among the methods is one of the criterion used to select data. Invariably, a goal has been to obtain as long a period of record as possible (Bonnin et al. 2006; Hogg et al. 1989). This is warranted in cases where the precipitation record is stationary, particularly since many design standards require recurrence intervals either near or beyond the length of the station record. However, the use of long, nonstationary precipitation records has the potential to yield precipitation-frequency estimates that are not representative of the current (or future) extreme rainfall climatology. For example, if a station's precipitation record exhibits a long-term increasing trend in extreme precipitation, inclusion of the earliest years may result in underestimation of the true present-day rainfall extremes.

A related data selection practice used in both the current U.S. and Canadian extreme rainfall atlases is to include data from any station at which a data record of at least 30 (19 in the case of Canada) years exists. Thus, a station that reported data, for example, from 1910 to 1940 would be included in the current analysis. Presumably this practice has an even greater potential to influence the biases associated with assuming stationarity, as data from more recent years are not available to temper the bias.

In this study, the influence of using different data record lengths drawn from trended rainfall series is quantified by comparing the resulting rainfall extremes with those given by a known stationary extreme rainfall distribution. In section 2, the generation of stochastic trended and stationary extreme rainfall series is discussed, as are the statistical tests used to identify differences among the series. In section 3 , the results are presented for various rainfall recurrence probabilities at a set of U.S. stations with disparate extreme rainfall climatologies. The paper concludes with some practical recommendations for optimizing the sample size used in extreme rainfall analyses on the basis of trended data records.

\section{Data and methods}

\section{a. Station extreme rainfall distributions}

Daily precipitation data from five stations in the Global Historical Climate Network (Menne et al. 2012) were obtained using the Applied Climate Information System (ACIS; DeGaetano et al. 2015). These stations were chosen to represent regions of the United States with a range of extreme rainfall characteristics. At least 107 years of data were available at each location (Table 1), with records extending through 2017.

The python "Imoments" package was used to fit the generalized extreme value (GEV) distribution to partialduration series (PDS) using the methods of Hosking (1990). Each PDS includes the $n$ highest independent daily rainfall totals in the station record, where $n$ approximates the number of years of valid data. Although not the only valid theoretical distribution for estimating extreme rainfall probabilities, the use of the GEV has been standard practice in prior extreme rainfall analyses (Papalexiou and Koutsoyiannis 2013). The probability density function (PDF) of the GEV distribution is given by

$$
f(x)=\left\{\begin{array}{ll}
\frac{1}{\sigma} \exp \left[-(1+k z)^{-1 / k}\right](1+k z)^{-1-(1 / k)} & k \neq 0 \\
\frac{1}{\sigma} \exp [-z-\exp (-z)] & k=0
\end{array},\right.
$$

where

$$
z=(x-\mu) / \sigma
$$


and $k, \sigma$, and $\mu$ are the shape, nonzero scale, and location parameters, respectively (Table 1); $x$ here is daily precipitation.

Subsequent analyses were conducted using open-source Scipy routines (http://www.scipy.org). Given the L-moments estimates for the GEV parameters, the Scipy "genextreme. ppf" method was used obtain the inverse cumulative distribution function from which specified percentiles were extracted (Fig. 1). To align with conventional extreme rainfall analyses, the 50th, 98th, and 99th percentiles were specifically examined, because these correspond to average annual recurrence probabilities of $50 \%, 2 \%$, and $1 \%$ (i.e., the 2-, 50-, and 100-yr storms) (Table 1).

Using observed station-based extreme rainfall series ensures that the theoretical distributions used in subsequent analyses are representative of the different regional precipitation regimes. For instance, the driest station, Phoenix, Arizona, is associated with the smallest location and shape parameters. Therefore, its PDF is the narrowest and is centered on the lowest precipitation amount (Fig. 1). The PDF for Miami, Florida, is the broadest and is shifted toward higher precipitation amounts (Fig. 1). The PDFs of the other stations are more similar, with location and scale increasing from Seattle, Washington, to Ithaca, New York, to Des Moines, Iowa (Fig. 1; Table 1). The shape parameters are negative and of similar magnitude at all stations.

\section{b. Resampled partial-duration series}

The fit GEV parameters (Table 1) also provide input to the Scipy "genextreme.rvs" method, which was used to generate a set of random PDS consistent with the stations' PDS. Given the GEV location, shape, and scale parameters, the genextreme.rvs routine returns a set of $n$ rainfall amounts consistent with the PDF. To account for the tendency of extreme rainfall amounts to be constrained by some theoretical physical upper limit (i.e., the probable maximum precipitation; Hershfield 1961, 1965; Douglas and Barros 2003), the magnitude of the random precipitation amounts selected from the continuous GEV distribution was limited to no more than three times the maximum value from the representative station's observed PDS. At each site, 1000 PDS $(n=$ $150 \mathrm{yr}$ ) were generated. Separate 1000 -member sets of shorter ( 90 and $60 \mathrm{yr}$ ) PDS were also generated using this approach. The various PDS lengths approximate the range of station record lengths used to develop extreme rainfall statistics from the U.S. climate record, with 150 years approximating an upper limit on PDS length. Few, if any, rainfall records exceed this length, with 90 and 60 years being more reflective of the available record length.

For each stationary resampled PDS, the L-moments method was used to generate new GEV parameters,

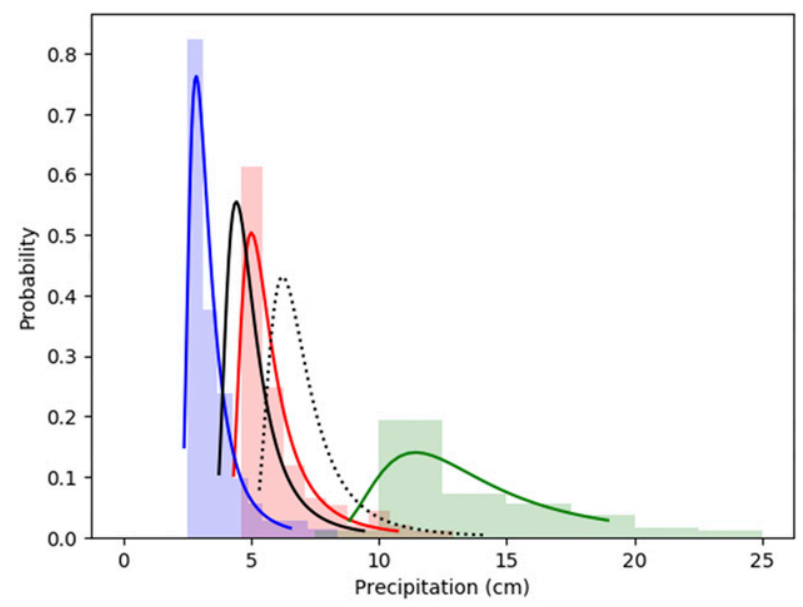

FIG. 1. GEV PDFs for Phoenix (blue), Seattle (solid black), Ithaca (red), Des Moines (dotted black), and Miami (green). Empirical PDS histograms for three stations are given by the shaded bars.

and, on the basis of these, the Scipy genextreme.ppf was used to extract the median, 98th, and 99th percentiles of the fit distributions. Hereinafter, these values are referred to as the 2-, 50-, and 100-yr storms to avoid confusion with other values that are expressed as percentages. From these 1000 synthetic series, a single PDS was randomly selected and retained as the base PDS. The choice represented the single PDS that would be used in a conventional station-data-based extreme value analysis. Sensitivity analyses showed that the subsequent results were not affected by different random choices. The full set of 1000 random PDS was used to construct $90 \%$ confidence intervals for the 2-, 50-, and 100-yr storms. These confidence intervals (CI) are designated base CI. The term "base" indicates that these PDS and CI correspond to those that would result from using the conventional analysis methods that assume stationarity. Therefore, the base values provide a benchmark for subsequent comparisons.

\section{RESAMPLED SERIES COMPARISONS}

In the same manner as described above, different sets of 1000 random stationary PDS with lengths ranging from 30 to 160 years in increments of 10 years were generated. Nonstationary test GEV distributions were also generated by imposing trends on the location and scale parameters consistent with the range of observed trends reported in the literature (Table 2). These stationary and nonstationary distributions are termed test series. Nonstationary $n$-yr test PDS were generated using the genextreme.rvs method by altering the location or scale parameters used in each year in a manner consistent with the published trends. 
TABLE 2. Extreme rainfall location and scale parameter trends reported in the literature.

\begin{tabular}{lcccc}
\hline \hline \multicolumn{1}{c}{ Region } & Period $(\mathrm{yr})$ & $\begin{array}{c}\text { Location } \\
\text { trend }\left(\% \mathrm{yr}^{-1}\right)\end{array}$ & $\begin{array}{c}\text { Scale trend } \\
\left(\% \mathrm{yr}^{-1}\right)\end{array}$ & \multicolumn{1}{c}{ Reference } \\
\hline Northern Europe & $1951-2010$ & 0.002 & -0.0007 & van den Besselaar et al. (2013) \\
Global land & $1990-2100$ & 0.0008 & 0.001 & Kharin and Zwiers (2005) \\
Oahu and Maui, HI & $1960-2010$ & -0.015 & -0.02 & Chen and Chu (2014) \\
Hawaii, HI & $1960-2010$ & 0.005 & 0.01 & Chen and Chu (2014) \\
Southern Brazil & $1896-2004$ & 0.01 & 0.01 & Pedron et al. (2017) \\
São Paulo, Brazil & $1933-2010$ & 0.004 & 0.004 & Silva Dias et al. (2013) \\
Northeastern United States & $1950-2007$ & 0.002 & 0.005 & DeGaetano (2009) \\
\hline
\end{tabular}

For each set of 1000 test PDS, four indices were computed:

1) the number of rejections of the null hypothesis $H_{o}-$ $\operatorname{PDS}_{\text {test }}(x)=\operatorname{PDS}_{\text {base }}(x)$, based on a KolmogorovSmirnov two-sample test (K-S2; Scholz and Stephens 1987), where $x$ indicates PDS length,

2) the proportion of the test distribution's $x$-yr storms that fell outside the corresponding base CI,

3) the $90 \%$ CI width of the test series, and

4) the number of $x$-yr storms that fell above and separately below the $\mathrm{CI}$.

In some cases, confidence interval widths were expressed relative to the magnitude of rainfall associated with their corresponding $x$-yr storm to facilitate comparison among stations and slopes. For instance, assume stations A's 100 -yr storm is $15 \pm 5 \mathrm{~cm}$ while that of station B is $30 \pm$ $10 \mathrm{~cm}$; in both cases, the relative confidence interval is 0.67 despite the actual confidence interval being 2 times as large at station $\mathrm{B}$.

In the case of K-S2 hypothesis test, the Scipy method "scipy.stats.ks_2samp" was used. This routine computes the Kolmogorov-Smirnov statistics on two independent samples drawn from a continuous distribution. Since the K-S2 test was run at the $\alpha=0.05$ level, the expectation is that $H_{o}$ would be falsely rejected in $5 \%$ of the 1000 trials when $\operatorname{PDS}_{\text {test }}(x)$ and $\operatorname{PDS}_{\text {base }}(x)$ are stationary, of equal length, and drawn from the same known distribution. This premise was tested by generating 1000 stationary base PDS series, and for each tallying the number of K-S2 rejections associated with 1000 different stationary test PDS of the same length drawn from the same distribution). There was a substantial range of false rejection percentages. However, in $90 \%$ of the cases, rejection rates fell between $2 \%$ and $20 \%$, regardless of PDS length or station. This resampled range guided subsequent analyses (i.e., only when more than $20 \%$ of the nullhypothesis tests were rejected was the PDS considered to be significantly different). The deviation from the expected false rejection rate appears to be an artifact of constraining the random PDS to values $<3$ times the maximum value in the observed PDS. The false rejection interval narrowed considerably when this constraint was removed, consistent with the KS-2 test's assumption of continuous data. Constructing the false rejection region in this manner also accounts for the

\section{Stationary pds of varying length}

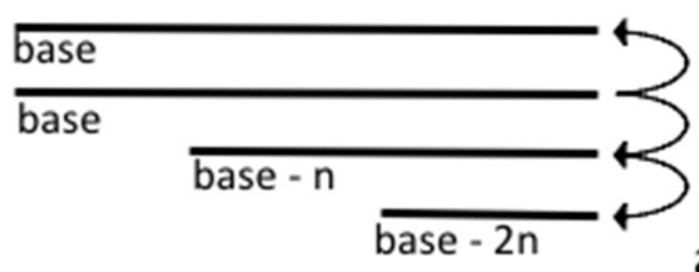

a
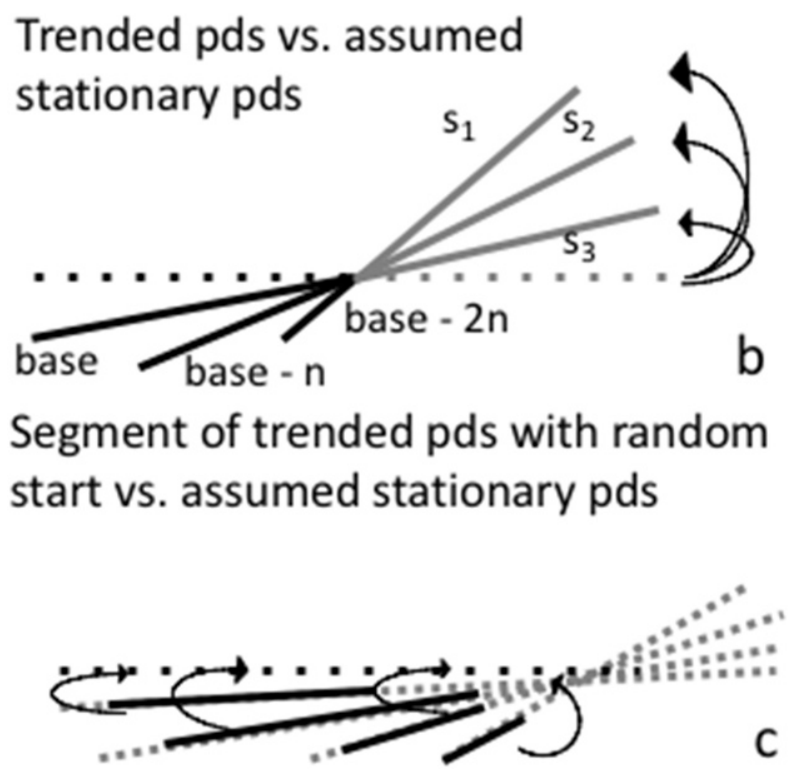

FIG. 2. Schematic diagram of the three cases tested: (a) comparison of stationary base series of varying length (base $-n$ ), (b) comparison of trended series (with different slopes $s$ and lengths $n$ ) and an assumed stationary base series, and (c) comparison of short trended series with random starting points and an assumed stationary base series. In (a), the vertical separation of the series is for clarity because these are drawn from the same distribution. The dotted line depicts the assumed stationary series that is given by the $k, \sigma$, and $\mu$ values fit to a trended observed record. 

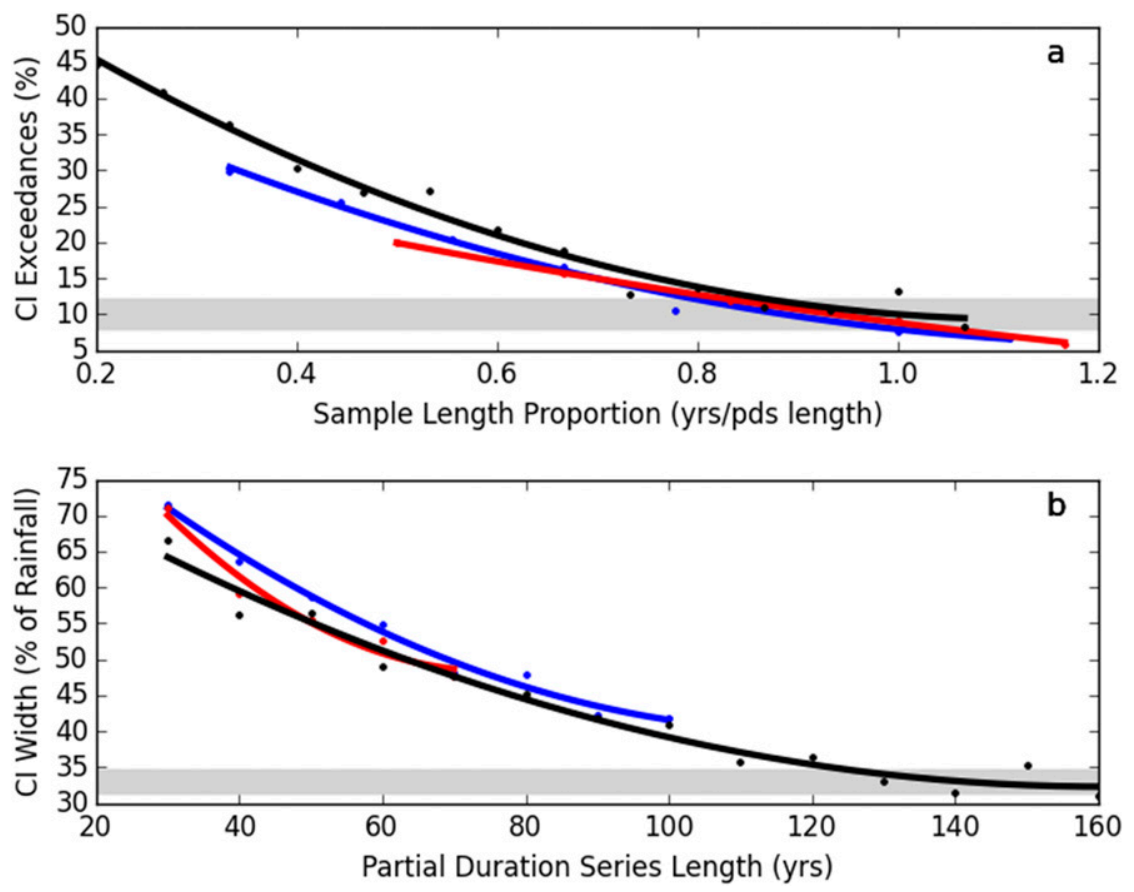

FIG. 3. (a) Percentage of PDS $_{\text {base }} 90 \%$ CI exceedances associated with subsamples of different lengths drawn from 150- (black), 90- (blue), and 60-yr (red) base series records. An $x$-axis value of 0.6 corresponds to a $90-\mathrm{yr}$ subsample from a 150 -yr base series or a $54-\mathrm{yr}$ subsample from a $90-\mathrm{yr}$ base series. (b) The $90 \%$ CI widths for PDS of various lengths expressed as a percentage of the computed rainfall extreme. In (a) and (b) solid lines are quadratic fits of the raw data values (dots). Shaded regions show the interdecile range of values from 1000 simulations.

possibility that the randomly selected base PDS was an outlier.

Three cases were specifically examined. These are illustrated schematically in Fig. 2. In case 1, stationary test PDS with lengths ranging from 30 to $n+10$ years in increments of 10 were compared with the $n$-yr $(n=150$, 90 , or 60) base distributions. These tests serve to 1 ) provide context for subsequent tests using trended series, 2) demonstrate how CI exceedances and width vary as a function of PDS length, and 3) assess the veracity of the resampling tests commonly used to construct extreme rainfall CIs.

In case 2 (Fig. 2b), trended test PDS of sequentially longer lengths were compared with those from stationary $n$-yr base distributions. It is not necessary to impose a trend on the base series, because its purpose is simply to establish a starting distribution based on $k, \sigma$, and $\mu$. In practice, even if the precipitation series is trended, extreme value analyses commonly proceed using nonvarying $k, \sigma$, and $\mu$ values that imply a stationary record (e.g., the dotted line in Figs. 2b). Practically, this set of analyses can be used to estimate the uncertainly in future extreme rainfall estimates that are based on past extreme rainfall data that were assumed to be stationary.
In conjunction with the results of the case 1 analyses, these results can be expected to provide guidance for establishing limits on the maximum length of record for use in extreme rainfall analyses under a changing climate. For instance, it may not be prudent to use a 100 -yr trended times series because the inclusion of the earlier nonrepresentative years could bias the extremes that represent the most recent part of the record.

In case 3 (Fig. 2c), PDS of varying length and starting at random points within a longer trended series were compared to the stationary base PDS. In extreme rainfall atlases (e.g., Bonnin et al. 2006; Hogg et al. 1989), it is a common practice to use rainfall records from currently inactive weather stations. As long as a time series with a sufficient data record length exists, an extreme rainfall analysis is conducted. This approach potentially exaggerates (relative to the scenario tested in case 2) the effect of assuming a stationary time series, because recent years that are more representative of the trended conditions are excluded from the analysis. Thus, the values of $k, \sigma$, and $\mu$ ascribed to this short, less-recent period are less representative of the current (and future) extreme rainfall distribution. 


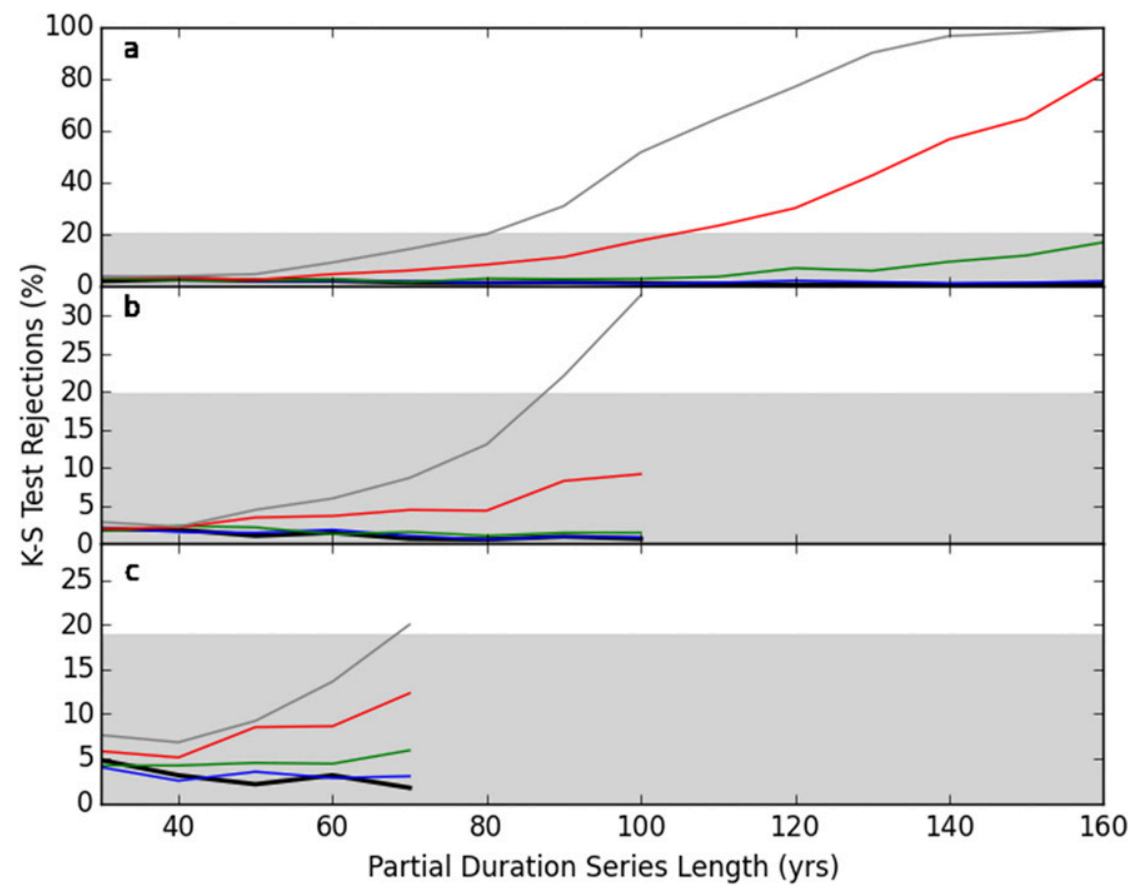

FIG. 4. Percentage of K-S2-test null-hypothesis rejections for PDS drawn from a GEV distribution with location parameter trend set to 0.000 (black), 0.002 (blue), 0.004 (green), 0.007 (red), and 0.01(gray). Partial-duration series length is set at (a) 150,(b) 90, and (c) $60 \mathrm{yr}$. The gray band is the $95 \%$ confidence range of K-S2-test rejections obtained from 1000 replications on the basis of stationary PDS of equal length.

\section{Results}

\section{a. Stationary time series}

Figure 3 a shows that for a stationary time series representing Ithaca, the proportion 50-yr CI exceedances decreases exponentially as the test PDS sample length approaches the length of the base PDS. For instance, the 50 -yr return periods computed from a 30 -yr subsample exceed base $15090 \%$ CI in nearly one-half of the cases. However, when the lengths of the test and base series are equal, estimates fall outside the $90 \% \mathrm{CI}$ in $10 \%$ of the cases as expected. When the analysis using equal base and test size (i.e., 1000 test PDS in comparison with the base PDS CI) is replicated 1000 times, $90 \%$ of the CI exceedances range from $8.8 \%$ to $11.9 \%$ (gray band). An equal percentage of these exceedances fall above and below the CI.

Figure $3 \mathrm{a}$ is representative of the other stations and return periods as well as shorter base PDS. This demonstrates that the resampling approach applied here and commonly used in operational extreme rainfall analyses, provides valid CIs regardless of PDS length. However, Fig. 3a also highlights that the use of a short PDS substantially increases the uncertainty in recurrence interval precipitation estimates relative to those based on a longer time series. Estimates based on a 30 -yr subset of a 150 -yr record fall outside the
150 -yr period's CI in nearly half of the cases. The $30-\mathrm{yr}$ subset exceeds the CI of the $90-\mathrm{yr}(60 \mathrm{yr})$ record in $30 \%$ $(20 \%)$ of the cases. Alone, this advocates for the conventional use of the longest stationary record possible.

Figure $3 \mathrm{~b}$ illustrates that, similar to CI exceedances, CI width (relative to rainfall amount) decreases with PDS length. Regardless of the length of the base PDS, 30-yr subsamples produce the widest confidence intervals (nearly $70 \%$ of the observed recurrence interval precipitation amount). This means that any 30-yr PDS, regardless of whether it is drawn from a 150-yr (black) or 60 -yr (red) base PDS, will give a CI width that is $70 \%$ of the fit rainfall extreme $(7 \mathrm{~cm}$ wide for a rainfall extreme of $10 \mathrm{~cm}, 14 \mathrm{~cm}$ wide for a $20-\mathrm{cm}$ extreme rainfall amount, etc.) CI width is approximately $50 \%$ of the observed recurrence interval precipitation amount for PDS subsamples of 60 years. The change in CI width tends to flatten for PDS lengths $>80$ years. Between $n=80$ and $n=160$, the CI width decreases from $45 \%$ to $30 \%$ of the recurrence period precipitation amount. This general pattern holds for the other stations and recurrence intervals.

\section{b. Trended location}

Using Ithaca as a representative example, Fig. 4 shows that PDS subsamples with a trend imposed on 


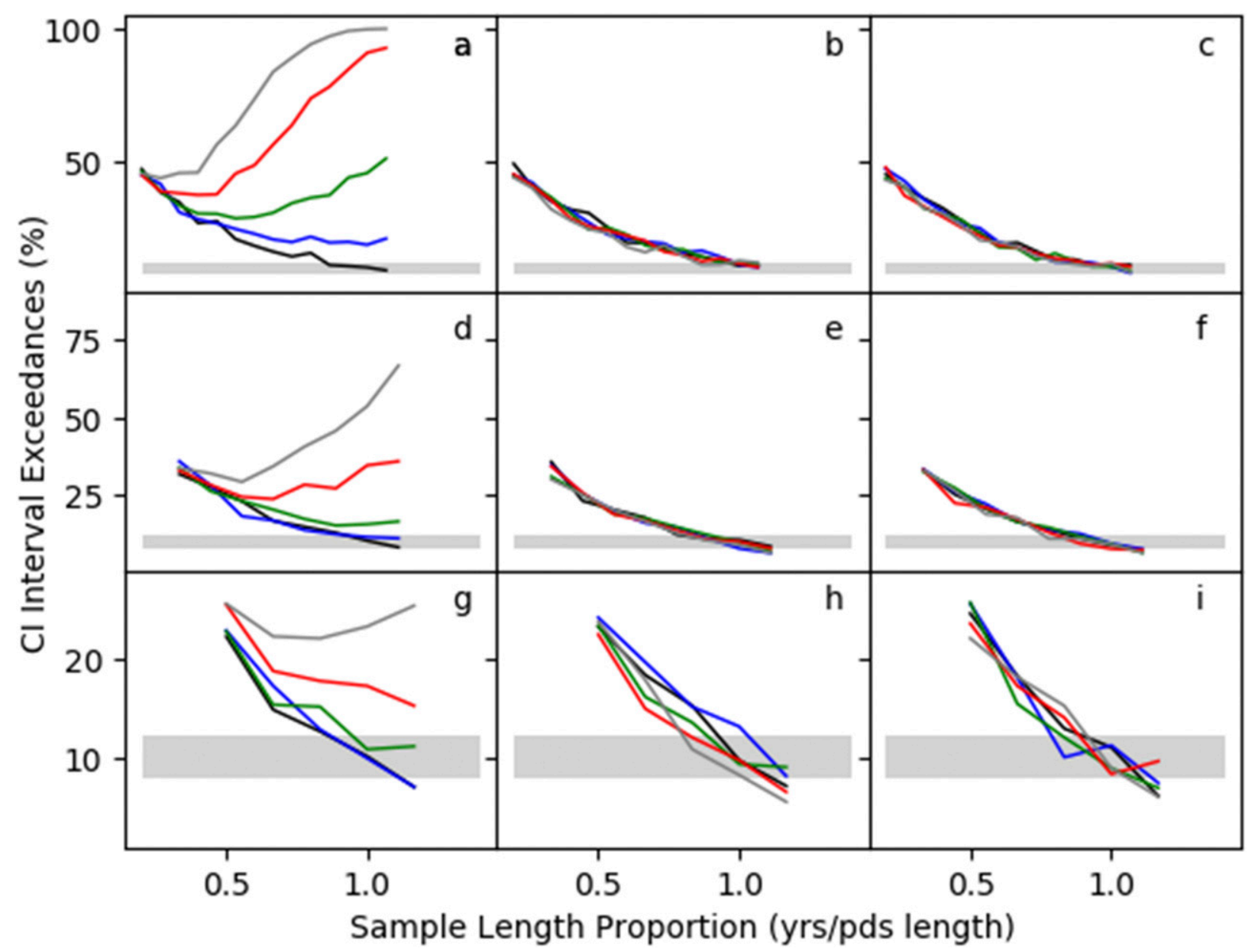

FIG. 5. Percentage of $90 \%$ CI exceedances for values of the (left) 2-, (center) 50-, and (right) 100-yr storm drawn from a GEV distribution with location parameter trend set to 0.000 (black), 0.002 (blue), 0.004 (green), 0.007 (red), and 0.01 (gray). Partial-duration series length is set at (a)-(c) 150, (d)-(f) 90, and (g)-(i) 60 years. The gray band is the $95 \%$ confidence range of CI exceedance percentages obtained from 1000 replications on the basis of stationary PDS of equal length.

the location parameter are different from those drawn from a stationary base series (Fig. 4a). The influence is greatest for the most pronounced trends and longest PDS, since the base series reflects only the earliest years of the trended record. For trends $\geq 0.01 \% \mathrm{yr}^{-1}$ the null hypothesis $\left(\mathrm{PDS}_{\text {test }}=\mathrm{PDS}_{\text {base }}\right)$ is rejected for trended PDS with lengths greater than 70-90 years, depending on the length assumed for PDS $_{\text {base }}$ (Fig. 4). The null hypothesis is also rejected for trends $>0.007 \% \mathrm{yr}^{-1}$ for trended $\mathrm{PDS}_{\text {test }}$ lengths greater than 100 years (Fig. 4a). In general, the number of K-S test rejections increases with larger location parameter trends and longer trended PDS. There is not a strong relationship between K-S rejections and the length of the stationary $\mathrm{PDS}_{\text {base }}$.

The differences suggested by the K-S test become more apparent when viewed in terms of return period CI exceedances (Fig. 5). For stationary series, CI exceedances are expected to decline with PDS length as in Fig. 3. This is the case for 100- and 50-yr storms, regardless of both the magnitude of location parameter trend and the length of the PDS used to define CI width. However, for the 2-yr storm, CI exceedances for the trended PDS never decline to the expected range centered on $10 \%$, but rather show marked increases, particularly for trends $>0.004 \% \mathrm{yr}^{-1}$ and $\mathrm{PDS}_{\text {test }}$ lengths of greater than 60 years (Figs. $5 \mathrm{a}, \mathrm{d}, \mathrm{g}$ ). There are also differences in the expected proportion of stationary CI exceedances as a function of location parameter trend, with more exceedances for larger trends. Confidence interval width is not affected by the magnitude of the location parameter trend (not shown). Rather, the CI width for both trended and stationary subseries declines as in Fig. $3 b$ as PDS length increases. 


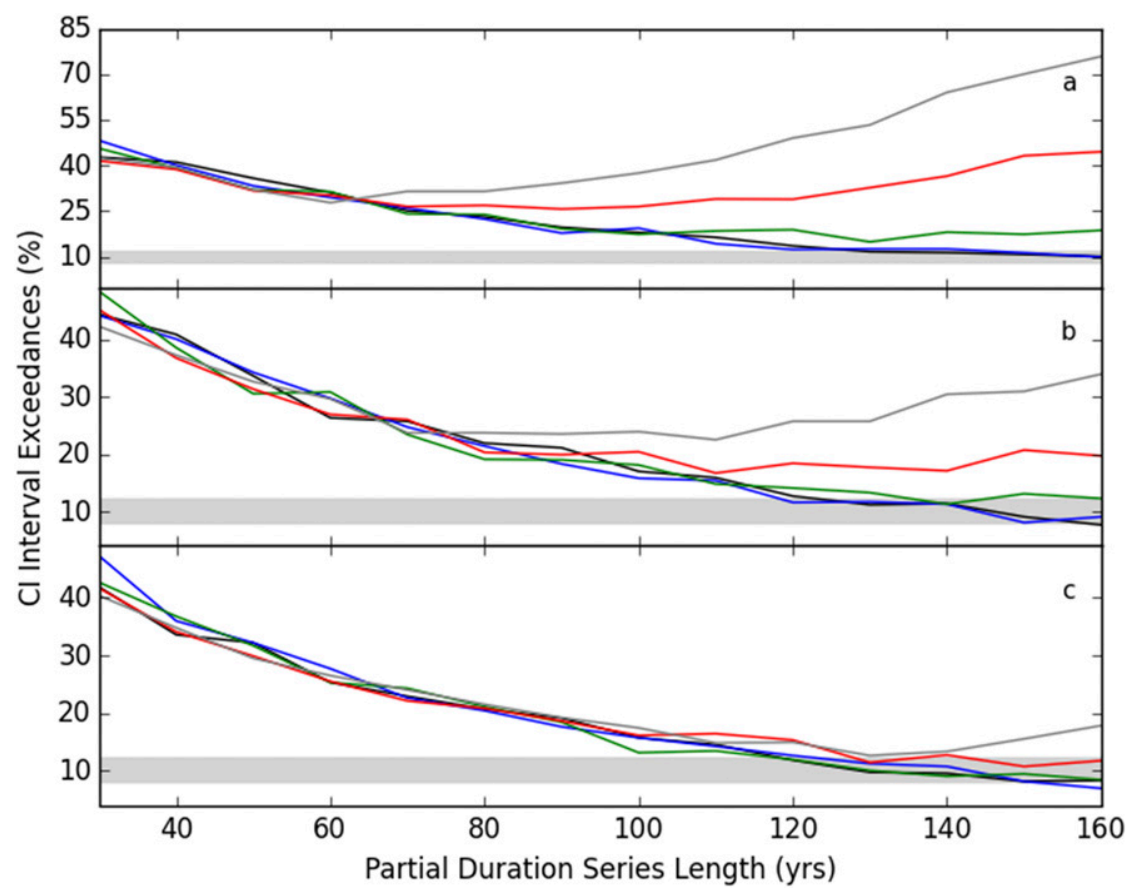

FIG. 6. Percentage of $90 \%$ CI exceedances for values of the (a) 5-, (b) 10-, and (c) 25-yr storm for PDS subsets of various lengths drawn from a GEV distribution with location parameter trend set to 0.000 (black), 0.002 (blue), 0.004 (green), 0.007 (red), and 0.01 (gray). The gray band is the $95 \%$ confidence range of CI exceedance percentages obtained from 1000 replications on the basis of a stationary 150-yr PDS.

For other intermediate recurrence periods commonly used in extreme rainfall analyses (i.e., 510 , and $25 \mathrm{yr}$ ), the influence of the trended location parameter is also apparent, but it is tempered as recurrence interval increases (Fig. 6). In the case of the 5-yr storm (Fig. 6a), more than $50 \%$ of the recurrence interval amounts calculated from a trended series fall outside the $90 \%$ CI of the stationary 150 -yr PDS for trends greater than $0.007 \% \mathrm{yr}^{-1}$. The number of 5-yr storm CI exceedances associated with different trends begins to diverge for trended PDS subseries greater than 70 years in length. This is also the case for the 10-yr storm (Fig. 6b). In the case of the 25-yr storm (Fig. 6c), CI exceedances behave similarly regardless of location parameter trend, except for the longest PDS ( $>140 \mathrm{yr})$, in which the percent of CI exceedances begins to increase.

Although the percentage of values falling outside the $90 \%$ CI range for recurrence periods of 25 years or greater is as expected, the magnitude of these values is biased. Considering the black dotted line in Fig. 7 corresponds to a stationary series, the percentage of values falling above the $90 \%$ CI upper bound is typically $50 \pm$ $10 \%$. However, for PDS lengths greater than 45 years, the majority of CI exceedances fall above the upper bound of the $90 \%$ CI. This is especially true for recurrence intervals of 50 years or less and for the longest
PDS lengths (Fig. 7, left and center columns), but it is also apparent when the 100-yr storm is considered (Fig. 7, right column). Values for the 2-yr storm computed from series with a minimal trend imposed on the location parameter are almost always higher than the upper bound of the CI given by an assumed stationary PDS. This has practical implications, as even for relatively short PDS lengths, extreme rainfall design criteria computed based upon the assumption of stationary will underestimate the true rainfall extreme more often than expected.

Last, Fig. 8 shows that the results presented for a GEV distribution representative of Ithaca also apply to a broad range of GEV distributions corresponding to U.S. locations with different extreme rainfall climatologies. For the 50-yr storm, CI exceedances exhibit analogous behavior among the stations (Figs. 8b,d,f,h). There is little difference in the percentage of expected CI exceedances for the different trends, with $10 \%$ of the rainfall estimates falling outside the $90 \%$ CI regardless of whether a trended or stationary location parameter is considered. However, as in Fig. 7, values from trended series predominantly exceed the stationary CI's upper bound.

The behavior of the 2-yr storm estimates for the other stations is similar to that for Ithaca (Figs. 8a,c,e,g). For PDS lengths greater than 45 years, the percentage of CI exceedances (which are almost always above the upper 


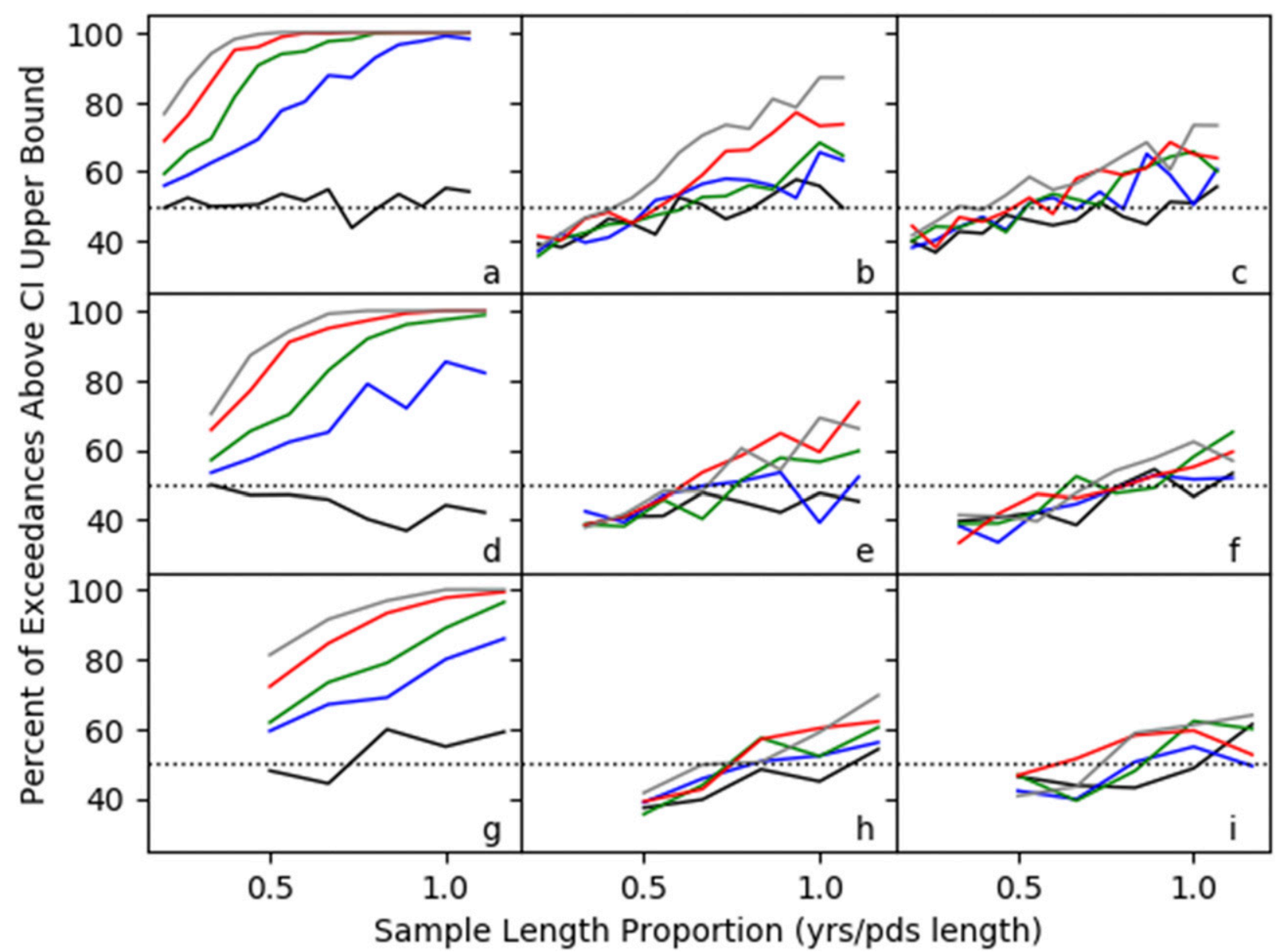

FIG. 7. Percentage of CI exceedances from Fig. 5 that fall above the upper CI bound (i.e., the 95th percentile). Panels and lines are as in Fig. 5, except for the dotted line at 50\%, which indicates percentage of exceedances that are expected to fall above the upper CI bound.

CI bound) increases, particularly for location parameter trends greater than $0.005 \% \mathrm{yr}^{-1}$. The most pronounced difference occurs for Miami, which has the largest location and scale parameters. Although the relationship between CI exceedances and PDS length and location parameter trend exhibits a similar pattern, the magnitude of the maximum exceedance percentages is about one-half as large as those that occur at the other stations (Fig. 8g).

\section{c. Trended scale}

While the literature also indicates nonstationary scale parameters, the simulations based on trended scale parameters did not result in any notable differences between the test and base PDS. In no cases could the null hypothesis be rejected at the $\alpha=0.05$ level. Likewise, the percentage of CI exceedances based on series with trended scale parameters could not be distinguished from that of a stationary series. These results were consistent among stations, recurrence intervals and PDS length.

\section{d. Random periods}

The previous analyses assumed that the location (scale) parameters of the stationary base series corresponded to the beginning year of the trended series. This simulates the potential biases associated with assuming the GEV parameters computed from the historical record of a currently operational station are applied in future years. Similar results were also obtained when the stationary parameters were associated with the ending year of the trended series, except the majority of trended exceedances were below the CI bound as opposed to above. In both cases, the stationary GEV parameters are comparable to GEV parameters associated with the first (last) portion of the trended series.

However, if the trended series $\left(\mathrm{PDS}_{\text {test }}\right)$ is a subset of a longer (also trended) PDS, the differences between the two series are more pronounced. This occurs because the GEV parameters associated with the full PDS can be quite different from those of the subset, particularly if 


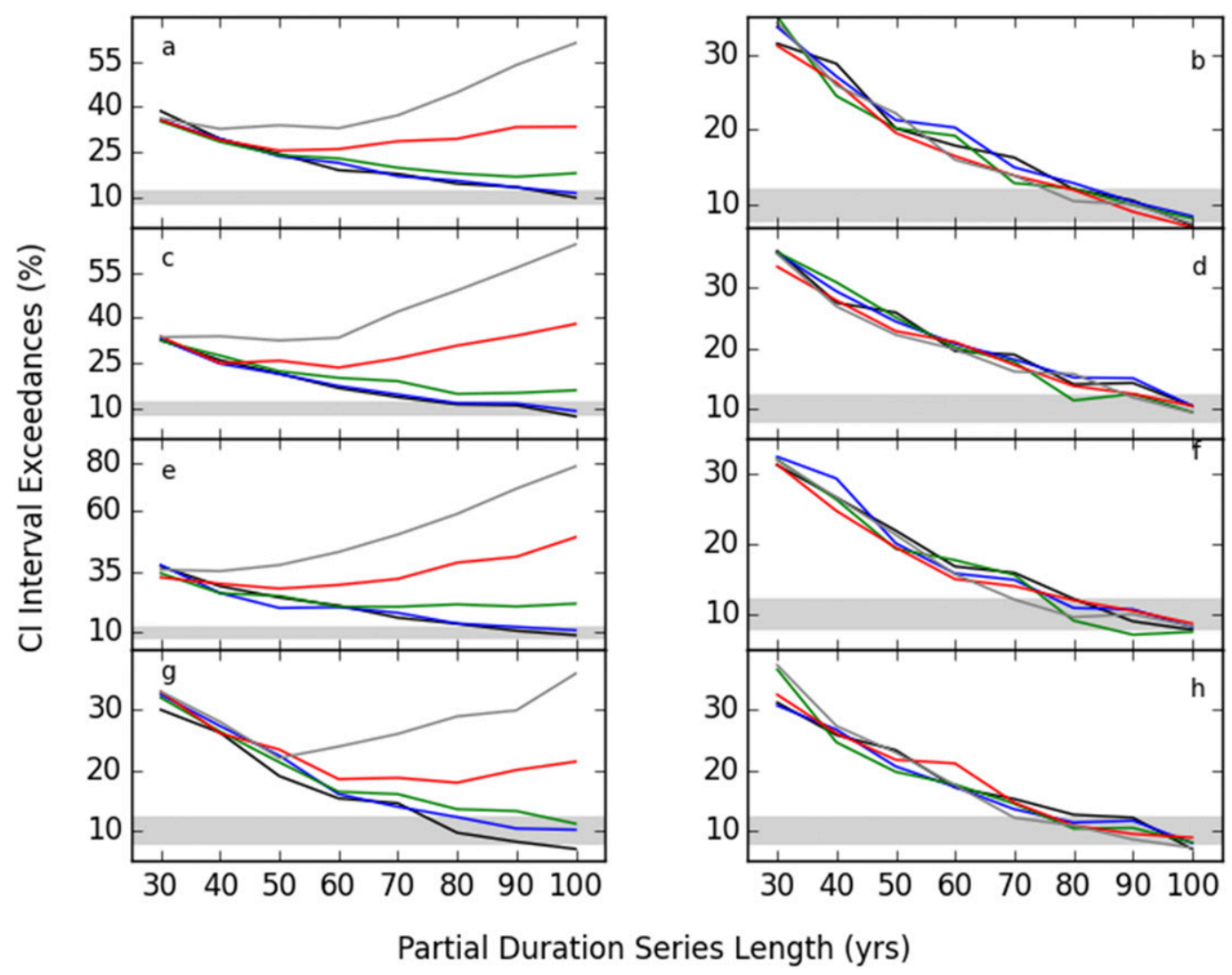

FIG. 8. As in Fig. 5 but using a 150-yr PDS and for the (left) 2- and (right) 50-yr storms from GEV distributions on the basis of data from (a), (b) Phoenix; (c), (d) Seattle; (e), (f) Des Moines; (g), (h) and Miami.

the subset is confined to the earliest part of the full PDS. Figure 9 shows that these differences are the greatest for short PDS subsets that commence early in the full PDS period. For location parameter trends as small as $0.004 \% \mathrm{yr}^{-1}$, significantly more 50 -yr storm rainfall estimates exceed the assumed stationary 90-yr PDS 90\% CI when 30-yr PDS starting early in the record (e.g., year 0 or year 25) are considered (Fig. 9). Similarly, 60-yr PDS subsets starting early in the record exceed the stationary $90 \%$ CI more often than expected when location parameter trend exceeds $0.004 \% \mathrm{yr}^{-1}$. When the PDS subset occurs in the later part of the 90-yr PDS (e.g., year 50 ), only the largest $0.01 \% \mathrm{yr}^{-1}$ trend result in a greaterthan-expected percentage of CI exceedances (Fig. 9).

\section{Summary and conclusions}

Collectively, the results indicate that for the range of GEV location parameter trends reported in the literature (Table 2), the assumption of nonstationarity in existing extreme rainfall analyses introduces the largest biases on the shortest recurrence intervals $(<25$ years $)$ and the longest data records ending with current data. For the most extreme events (e.g., those associated with recurrence intervals $>50$ years), the influence is confined to trends $\geq 0.007 \% \mathrm{yr}^{-1}$ at stations with very long PDS ( $>100$ years ending with current observations). Note, however, that for smaller trends and shorter record lengths, although the percentage of rainfall extremes that exceed the stationary series $90 \% \mathrm{CI}$ is no different than expected, those exceedances are overwhelmingly associated with higher rainfall amounts. The findings are consistent across locations exhibiting a range of extreme rainfall characteristics.

From a practical standpoint, these results suggest that future extreme rainfall analyses should implement a preliminary screening step to test and quantify the trend in the fit distribution's location parameter to consider whether the use of data records exceeding 70 years in length is warranted. In cases where the trend is present and exceeds $0.005 \% \mathrm{yr}^{-1}$, the data record should be truncated to no more than the most recent 70 years. This step is particularly important for applications that depend on the accuracy of the 2-yr recurrence interval value, such as urban stormwater design (ASCE 2006). 


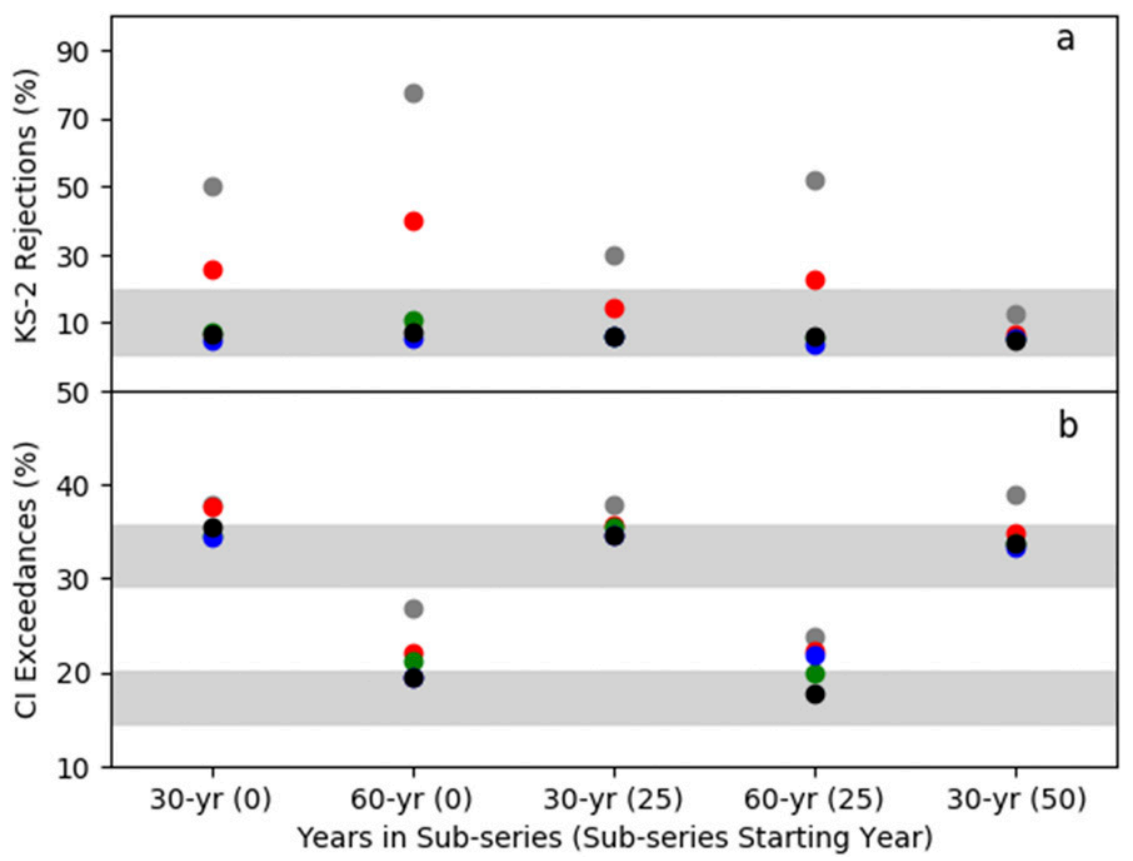

FIG. 9. Percentage of (a) K-S2-test null-hypothesis rejections and (b) $90 \%$ CI exceedances for the 50-yr storm for $n$-yr PDS subsets drawn from a GEV distribution representative of Seattle with location parameter trend set to 0.000 (black), 0.002 (blue), 0.004 (green), 0.007 (red), and 0.01(gray) relative to a 90-yr PDS with the same trend. The number in the parentheses indicates that starting point of the subseries within the 90-yr PDS. The gray band is the $95 \%$ confidence range of K-S2-test rejections and exceedances obtained from 1000 replications on the basis of a stationary 90 -yr PDS. In (b), the uppermost confidence range corresponds to 30 -yr subseries and the lower band to 60 -yr subseries.

For applications that rely on longer return frequencies (e.g., 50 or $100 \mathrm{yr}$ ), a trend of $0.01 \% \mathrm{yr}^{-1}$ or less appears to have little influence on the calculated rainfall extremes, regardless of record length, provided the record includes the most recently observed data. Thus, in these cases it appears that the conventional practice of using the longest available data record is still advisable. All recurrence interval rainfall estimates (including those for 50 and 100 years) are adversely affected by the use of short $(<60 \mathrm{yr})$ data segments that end more than $20-30$ years before the current date. The use of such data segments should be avoided, especially at stations within regions exhibiting a consistent trend in extreme value distribution location parameters.

The proposed initial screening step might also examine the PDS for decadal-scale periodicities. Such behavior might bias the computed return periods in a manner similar to the trends analyzed in this study. Presumably cycles that are short relative to the available PDS length will have little effect on the shortest return periods. However, cycles having longer periods could manifest themselves as trends. Such cycles would be difficult to detect given the length of most station records. An interesting extension of this work would be to use a similar methodology to examine the interaction of cycle frequencies and record length on extreme rainfall recurrence. Such an analysis should also account for the possibility of a both a trend and decadal-scale cycle in the extreme rainfall record.

Acknowledgments. This work was supported by the New York State Energy Research and Development Authority (NYSERDA) under Contract 28257 and by National Oceanic and Atmospheric Administration Contract AB-133E-16-CQ-0025.

\section{REFERENCES}

ASCE, 2006: Standard Guidelines for the Design (45-05), Installation (46-05), and Operation and Maintenance (47-05) of Urban Stormwater Systems. American Society of Civil Engineers, 120 pp., https://doi.org/10.1061/9780784408063.

Bonnin, G. M., D. Martin, B. Lin, T. Parzybok, M. Yekta, and D. Riley, 2006: Precipitation-frequency atlas of the United States. Vol. 2, NOAA Atlas 14, 65 pp. + appendixes, http:// www.nws.noaa.gov/oh/hdsc/PF_documents/Atlas14_Volume2.pdf.

Chen, Y. R., and P.-S. Chu, 2014: Trends in precipitation extremes and return levels in the Hawaiian Islands under a changing climate. Int. J. Climatol., 34, 3913-3925, https://doi.org/ 10.1002/joc. 3950 . 
Cheng, L., A. AghaKouchak, E. Gilleland, and R. W. Katz, 2014: Non-stationary extreme value analysis in a changing climate. Climatic Change, 127, 353-369, https://doi.org/10.1007/ s10584-014-1254-5.

DeGaetano, A. T., 2009: Time-dependent changes in extremeprecipitation return-period amounts in the continental United States. J. Appl. Meteor. Climatol., 48, 2086-2099, https://doi.org/ 10.1175/2009JAMC2179.1.

— , and C. M. Castellano, 2017: Future projections of extreme precipitation intensity-duration-frequency curves for climate adaptation planning in New York State. Climate Serv., 5, 23-35, https://doi.org/10.1016/j.cliser.2017.03.003.

— W. Noon, and K. L. Eggleston, 2015: Efficient access to climate products using ACIS web services. Bull. Amer. Meteor. Soc., 96, 173-180, https://doi.org/10.1175/BAMS-D-13-00032.1.

Donat, M. G., A. L. Lowry, L. V. Alexander, P. A. O'Gorman, and N. Maher, 2016: More extreme precipitation in the world's dry and wet regions. Nat. Climate Change, 6, 508, https://doi.org/ 10.1038/nclimate2941.

Douglas, E. M., and A. P. Barros, 2003: Probable maximum precipitation estimation using multifractals: Application in the eastern United States. J. Hydrometeor., 4, 1012-1024, https:// doi.org/10.1175/1525-7541(2003)004<1012:PMPEUM>2.0.CO;2.

Groisman, P. Ya., R. W. Knight, and T. R. Karl, 2012: Changes in intense precipitation over the central United States. J. Hydrometeor., 13, 47-66, https://doi.org/10.1175/JHM-D-11-039.1.

Hailegeorgis, T. T., and K. Alfredsen, 2017: Analyses of extreme precipitation and runoff events including uncertainties and reliability in design and management of urban water infrastructure. J. Hydrol., 544, 290-305, https://doi.org/10.1016/ j.jhydrol.2016.11.037.

Heineman, M., 2012: Trends in precipitation maxima at U.S. Historical Climatology Network stations 1893-2010. Proc. World Environmental and Water Resources Congress 2012: Crossing Boundaries, American Society of Civil Engineers, 2003-2012, https://doi.org/10.1061/9780784412312.201.

Hershfield, D. M., 1961: Rainfall frequency atlas of the United States. U.S. Weather Bureau Tech. Paper 40, 65 pp., http:// www.nws.noaa.gov/oh/hdsc/PF_documents/TechnicalPaper_ No40.pdf.

_- 1965: Method for estimating probable maximum rainfall. J. Amer. Water Works Assoc., 57, 965-972, https://doi.org/ 10.1002/j.1551-8833.1965.tb01486.x.

Hogg, W. D., D. A. Carr, and B. Routledge, 1989: Rainfall intensity-duration frequency values for Canadian locations. Atmospheric Environment Service Rep. CLI-1-89, 17 pp.

Hosking, J. R. M., 1990: L-moments: Analysis and estimation of distributions using linear combinations of order statistics. J. Roy. Meteor. Soc., 52, 105-124.

Huff, F. A., and J. R. Angel, 1992: Rainfall frequency atlas of the Midwest. Illinois State Water Survey Bull. 71, 148 pp., https:// www.nrcs.usda.gov/Internet/FSE_DOCUMENTS/nrcs141p2_ 024033.pdf.

IPCC, 2014: Climate Change 2014: Synthesis Report. R. K. Pachauri and L. A. Meyer, Eds., Cambridge University Press, 151 pp., https://www.ipcc.ch/pdf/assessment-report/ar5/syr/SYR_AR5_ FINAL_full.pdf.

Katz, R. W., M. B. Parlange, and P. Naveau, 2002: Statistics of extremes in hydrology. Adv. Water Resour., 25, 1287-1304, https://doi.org/10.1016/S0309-1708(02)00056-8.
Kharin, V. V., and F. Zwiers, 2005: Estimating extremes in transient climate change simulations. J. Climate, 18, 1156-1173, https://doi.org/10.1175/JCLI3320.1.

Kunkel, K. E., 2003: North American trends in extreme precipitation. Nat. Hazards, 29, 291-305, https://doi.org/10.1023/ A:1023694115864.

—, K. Andsager, and D. R. Easterling, 1999: Long-term trends in extreme precipitation events over the conterminous United States and Canada. J. Climate, 12, 2515-2527, https://doi.org/ 10.1175/1520-0442(1999)012<2515:LTTIEP>2.0.CO;2.

Menne, M. J., I. Durre, R. S. Vose, B. E. Gleason, and T. G. Houston, 2012: An overview of the Global Historical Climatology Network-Daily database. J. Atmos. Oceanic Technol., 29, 897-910, https://doi.org/10.1175/JTECH-D-11-00103.1.

Ning, L., E. E. Riddle, and R. S. Bradley, 2015: Projected changes in climate extremes over the northeastern United States. J. Climate, 28, 3289-3310, https://doi.org/10.1175/JCLI-D-1400150.1 .

Papalexiou, S. M., and D. Koutsoyiannis, 2013: Battle of extreme value distributions: A global survey on extreme daily rainfall. Water Resour. Res., 49, 187-201, https://doi.org/10.1029/ 2012 WR012557.

Pedron, I. T., M. A. Silva-Dias, S. de Paula Dias, L. Carvalho, and E. D. Freitas, 2017: Trends and variability in extremes of precipitation in Curitiba-southern Brazil. Int. J. Climatol., 37, 1250-1264, https://doi.org/10.1002/joc.4773.

Perica, S., and Coauthors, 2013: Precipitation-frequency atlas of the United States. Vol. 9, NOAA Atlas 14, 36 pp. + appendixes, http://www.nws.noaa.gov/oh/hdsc/PF_documents/Atlas14_ Volume9.pdf.

Rodríguez, R., X. Navarro, M. C. Casas, J. Ribalaygua, B. Russo, L. Pouget, and A. Redaño, 2014: Influence of climate change on IDF curves for the metropolitan area of Barcelona (Spain). Int. J. Climatol., 34, 643-654, https://doi.org/10.1002/joc.3712.

Scholz, F. W., and M. A. Stephens, 1987: $K$-sample AndersonDarling tests. J. Amer. Stat. Assoc., 82, 918-924.

Silva Dias, M. A. F., J. Dias, L. M. V. Carvalho, E. D. Freitas, and P. L. Dias, 2013: Changes in extreme daily rainfall for São Paulo, Brazil. Climatic Change, 116, 705-722, https://doi.org/ 10.1007/s10584-012-0504-7.

Sun, Q., C. Miao, and Q. Duan, 2016: Extreme climate events and agricultural climate indices in China: CMIP5 model evaluation and projections. Int. J. Climatol., 36, 43-61, https://doi.org/ 10.1002/joc. 4328 .

van den Besselaar, E. J. M., A. M. G. Klein Tank, and T. A. Buishand, 2013: Trends in European precipitation extremes over 1951-2010. Int. J. Climatol., 33, 2682-2689, https://doi.org/ 10.1002/joc.3619.

van der Pol, T. D., E. C. van Ierland, S. Gabbert, H. P. Weikard, and E. M. T. Hendrix, 2015: Impacts of rainfall variability and expected rainfall changes on cost-effective adaptation of water systems to climate change. J. Environ. Manage., 154, 40-47, https://doi.org/10.1016/j.jenvman.2015.02.016.

Wang, X., G. Huang, J. Liu, Z. Li, and S. Zhao, 2015: Ensemble projections of regional climatic changes over Ontario, Canada. J. Climate, 28, 7327-7346, https://doi.org/10.1175/JCLI-D-150185.1.

Wilks, D. S., and R. P. Cember, 1993: Atlas of precipitation extremes for the northeastern United States and southeastern Canada. Northeast Regional Climate Center Research Publ. RR 93-5, 40 pp. 